\title{
Generalized Spectral Signatures of Electron Fractionalization in Quasi-One and -Two Dimensional Molybdenum Bronzes and Superconducting Cuprates
}

\author{
G.-H. Gweon ${ }^{\dagger}{ }^{1}$ J. W. Allen, ${ }^{1}$ and J. D. Denlinger ${ }^{2}$ \\ ${ }^{1}$ Randall Laboratory of Physics, University of Michigan, 500 E. University, Ann Arbor, MI 48109 \\ ${ }^{2}$ Advanced Light Source, Lawrence Berkeley National Laboratory, Berkeley, CA 94720
}

(Dated: October 31, 2018)

\begin{abstract}
We establish the quasi-one-dimensional Li purple bronze as a photoemission paradigm of Luttinger liquid behavior. We also show that generalized signatures of electron fractionalization are present in the angle resolved photoemission spectra for quasi-two-dimensional purple bronzes and certain cuprates. An important component of our analysis for the quasi-two-dimensional systems is the proposal of a "melted holon" scenario for the k-independent background that accompanies but does not interact with the peaks that disperse to define the Fermi surface.

PACS numbers: 71.10.Pm, 71.20.-b, 79.60.-i
\end{abstract}

\section{INTRODUCTION}

In the Landau Fermi-Liquid (FL) theory [1] of interacting electrons, low energy single-particle, i.e. electron addition and removal, excitations behave like free electrons except for enhanced mass and therefore are termed Landau "quasi-particles." The electron in this generalized sense retains its status as a fundamental particle. One paradigm for non-FL (NFL) behavior is the electron fractionalization that occurs in the so-called Luttinger liquid (LL), as defined 2 by the phenomenological applicability of the Tomonaga-Luttinger (TL) model [3, 4] of interacting electrons in one-dimension. In this new state, electron-like single-particle eigenstates no longer exist, and an electron/hole excitation propagates only as a continuum of collective density waves. In this sense, the electron is "fractionalized." [5, 6, 7] Two key features of LL fractionalization are power law behaviors of correlation functions, characterized by an anomalous dimension $(\alpha)$, and the complete separation of spin and charge degrees of freedom into density waves dispersing with different velocities $\mathrm{v}_{\mathrm{s}}, \mathrm{v}_{\mathrm{c}}$. The Luther-Emery (LE) model [8] differs from the TL model by an additional interaction term that causes a gap in the spin excitation spectrum. In this paper we focus on the single particle spectral function, which has characteristic features that are much different from that of a FL. This spectral function can be measured in angle resolved photoemission spectroscopy (ARPES).

In spite of many efforts on ARPES in quasi-low-d systems 9, 10, 11], there remains much skepticism as to the relevance of fractionalization to these spectra. First, the TL model is strictly one-dimensional. It is then a serious question whether actual quasi-1-d materials with their underlying higher dimensionality could display TL (or LE) behavior and beyond that, whether such behavior could be found even in quasi-2-d materials such as the superconducting cuprates, as has been proposed [5, 6] in various scenarios. Second, the ARPES spectra of low di- mensional materials generally differ in various ways from the simple model spectra. Nonetheless the experimental spectra display definite NFL attributes. Our stance here is that the simple models correctly show generic possibilities while lacking one or more of the elements needed for describing actual data. Treating all the important elements, e.g. multiple bands, the full Coulomb interaction, electron-phonon interactions, and higher dimensional couplings, all on an equal footing, is beyond current theory. But we surmise that fractionalization may be the correct physics underlying the more complex reality, even for some materials with dimensionality greater than one.

In this paper we adduce strong evidence supporting such a hypothesis. We have obtained over time 11, 12, 13, 14] increasingly more detailed ARPES spectra of the quasi-1-d metal $\mathrm{Li}_{0.9} \mathrm{Mo}_{6} \mathrm{O}_{17}$ ( $\mathrm{Li}$ "purple bronze"). The 1-d Fermi surface (FS) found in these studies 12 is shown in panel (a) of Fig. 1] Here we show that, with the exception of one aspect that is nonetheless of definite NFL character, the dispersing lineshapes defining the ARPES FS are well described by finite temperature TL model theoretical spectra 15. This material is thus an LL ARPES paradigm. We also introduce generalized signatures of electron fractionalization extracted from the TL model and related thinking. Presentation of these signatures provides the basic organization of the paper and we show that they are present in our ARPES spectra of several low dimensional bronzes 19], including those of quasi-1-d $\mathrm{K}_{0.3} \mathrm{MoO}_{3}$ (the $\mathrm{K}$ "blue bronze") and of quasi2-d $\mathrm{NaMo}_{6} \mathrm{O}_{17}$ (the Na "purple bronze"). The Na purple bronze is significant here as a bridge material between 1-d and 2-d because of its "hidden 1-d" FS 20] arising from three weakly coupled 1 -d chains that are mutually oriented at 120 degrees. We have previously measured its FS 21] by ARPES, as reproduced in panel (b) of Fig. [1] and in this paper we show that the associated ARPES spectra display generalized fractionalization signatures that are also present in the ARPES spectra of the quasi-2d superconducting cuprate $\mathrm{Bi}_{2} \mathrm{Sr}_{2} \mathrm{CaCu}_{2} \mathrm{O}_{8+\delta}$ (Bi2212) 


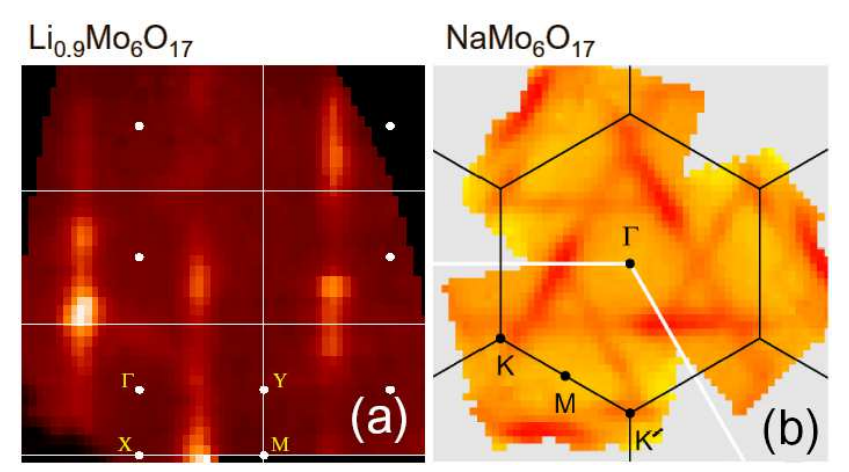

FIG. 1: ARPES FS maps for (a) quasi-1-d Li purple bronze [12] and (b) quasi-2-d (hidden 1-d) Na purple bronze 21].

[22, 23]. An important component of the analysis is to identify the effect of charge disorder on the LL spectra.

We are concerned here only with normal state line shapes above phase transitions to ordered states, e.g. charge density wave (CDW), spin density wave (SDW), or superconductivity (SC). Whereas the ordered state must entail higher dimensionality the normal state is expected to display quasi-1-d fractionalization behavior 24]. In this connection we note that models with electron-phonon interactions but no explicit electron-electron interactions show above $T_{C D W}$ a CDWfluctuation-induced pseudo-gapped NFL metallic state [25], which, we postulate, might also admit of a fractionalization description. The phase transitions of our materials occur at $24 \mathrm{~K}$ (Li purple bronze: order unknown but not CDW/SDW 11, 12]), $180 \mathrm{~K}$ (K blue bronze: CDW), $80 \mathrm{~K}$ (Na purple bronze: CDW), and $90 \mathrm{~K}$ (Bi2212: SC). X-ray scattering [16] due to CDW or SDW formation has never been found in the Li purple bronze and its optical conductivity [17] shows no gap down to $1 \mathrm{meV}$. It is however a SC below $1.9 \mathrm{~K}$ [18]. For the K blue and Na purple bronzes CDW fluctuation effects above $T_{C D W}$ have been identified in X-ray diffraction up to room temperature [19].

Throughout this paper, a single but typical ARPES data set is discussed for each material, plus data (Figs. 2(b-d)) taken for bronzes with an angle-integrated VG ESCALAB II spectrometer. The ARPES data were taken along 1-d chain axes for bronzes, and along the $(\pi, \pi)$ direction, i.e. the diagonal of the 2-d Brillouin zone (BZ) for Bi2212 22]. All of the data presented are already published [11, 14], with two exceptions (Figs. 2(b) and [3(d) [26]). In the following, we give values of the experimental energy resolution $\Delta E$ (in FWHM) and the full angular width $\Delta \theta$, where it becomes relevant to do so.

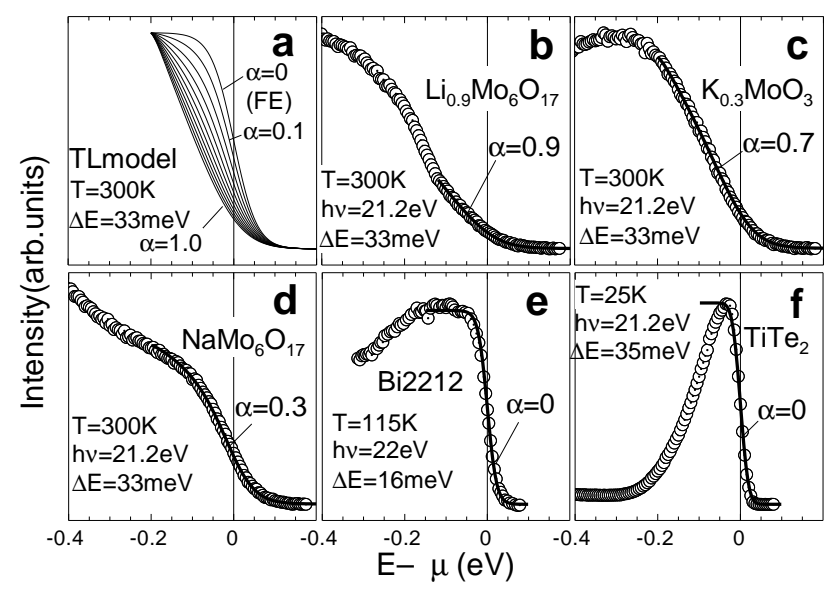

FIG. 2: Theoretical 15] and experimental angle-summed spectral functions. Best-fit curves from panel (a), with modified $\Delta E$ and $T$ values where appropriate, are shown as lines.

\section{GENERALIZED FRACTIONALIZATION SIGNATURES}

1. No Fermi Edge in Angle-Summed Spectrum Electron (or hole) fractionalization in the LL leads to a strong and surprisingly counter-intuitive suppression of the k-summed spectral function $\rho(\omega)$ at the chemical potential $(\mu)$, vanishing as a power law $|\omega-\mu|^{\alpha}$ at $T=0$. This is contrasted with the Fermi edge (FE) line shape of an FL, given by the Fermi-Dirac function. $\rho(\omega)$, broadened by $\Delta E$, is measured by the angle-summed ARPES and experimental data hinting at non-zero $\alpha$ have been known for some time [9].

We compare various angle-summed spectra in Fig. 2 with theoretical line shapes. As an experimental FL reference [27, 28, 29], we include the angle-sum of the $\mathrm{TiTe}_{2}$ ARPES data [27]. For comparison to the 1-d TL lineshapes, our angle integrated spectra of the quasi-1-d and hidden 1-d bronzes are effectively 1-d sums and for Bi2212 we sum ARPES spectra 22] along a line normal to the FS. One immediately notices a qualitative difference between the smooth onsets at $\mu$ of the quasi-1-d metals, definitely non-FL, and the more abrupt edges of the quasi-2-d metals, all resembling a FE. However, fits to the theoretical line shapes of panel (a) give $\alpha$ values that require more refined thinking: 0.9 (Li purple), 0.7 (K blue), 0.3 (Na purple), and 0 (Bi2212, $\mathrm{TiTe}_{2}$ ). Two new findings, enabled by the use here of the recent finite $T$ spectral function theory [15] of the TL model, are significant for the discussion below: a finite $\alpha$ for the quasi2- $d \mathrm{Na}$ purple bronze and an $\alpha$ value increased from that (0.6) obtained 12$]$ previously by applying a $\mathrm{T}=0$ theory to the same data for the Li purple bronze. The FE line shape for Bi2212 is in apparent contradiction to its nonFL properties [5, 6]. Along this particular line of $\mathbf{k}$-space [30] it is possible that the $\alpha$ value is so small that the 
spectrum is essentially the same as an FE for the given $T$ and $\Delta E$. But the second signature along this line, presented below, yields a moderately large $\alpha$ value, making it also possible that instead the energy range of the power law behavior is so small that it is completely masked by $T$ and $\Delta E$. Indeed theoretical work shows that such a small energy range, on the order of $10 \mathrm{meV}$, can occur for the 1-d Hubbard model [31]. A Hubbard model is likely for cuprates but not for the Mo bronzes whose $4 \mathrm{~d}$ orbitals are less localized.

Some comments are in order for the fits of Fig. 2 First, the TL model used here could be replaced by any theory which yields a power law spectrum at $T=0$ and obeys quantum critical scaling at finite $T$. Second, the smallness $(\Delta \approx 20 \mathrm{meV})$ of the spin pseudo-gap 32., which implicates the LE model for the blue bronze [10], justifies its neglect here. More specifically, in this paper we limit ourselves to the case $\max (T, \Delta E)>\Delta(T)$, where $\Delta(T)$ is any pseudo-gap. Third, the TL model assumes a constant one-electron density of states (DOS). Thus the $\alpha$ values obtained by the fit potentially have some un-quantified errors and so for the Na purple bronze we have verified explicitly that an FL line shape with a sloped DOS does not produce a good fit. Fourth, the energy range of the fit is determined a posteriori by the fit, and is marked by the energy range of the lines, except for $\mathrm{TiTe}_{2}$, for which only the FE width is fit because its unusually small band width 27] causes the strong drop of intensity as soon as the binding energy becomes bigger than the FE width. We note, in particular, that the fit range for the Li purple bronze is binding energy $<0.12 \mathrm{eV}$, at which point two non- $\mu$-crossing bands [11, 14] start to contribute and give rise to the break observed in the line shape of panel (b).

2. Anderson-Ren Line Shape This is another signature that we associate with $\alpha$. Anderson and Ren (AR) proposed [33] an empirical visualization scheme for the Bi2212 ARPES line shapes along the diagonal [30] of the 2-d BZ. In this view, the line shape is given by a common power law tail relative to $\mu$, with exponent $\alpha-1$, for binding energies greater than the peak position, and a straight line fall to $\mu$ for energies less than the peak position. Fig. 3 shows the dispersing line shapes of Bi2212 and of the bronzes, plotted with intensity scaling to show the remarkable result that the AR line shape is observed in all of them. In contrast, we find that the FL ARPES line shapes for the $\mathrm{Ti} 3 \mathrm{~d}$ band of $\mathrm{TiTe}_{2}$ [27] and also for a Mo surface state 35] do not follow the AR line shape.

We examine next the relationship between the exponents from the AR line shape and from the $\mu$ onset in angle-summed spectra. The plots in Fig. 3] show that the AR line shape does not hold when $\mathbf{k}$ approaches $\mathbf{k}_{\mathrm{F}}$. Therefore, the AR line shape, as identified here, is a high energy and high momentum behavior. Nonetheless, taking the $\mathrm{AR}$ connection to $\alpha$, we get $\alpha$ values of 0.4 (Bi2212), 0.2 (K blue), 0.7 (Li purple), and 0.4 (Na

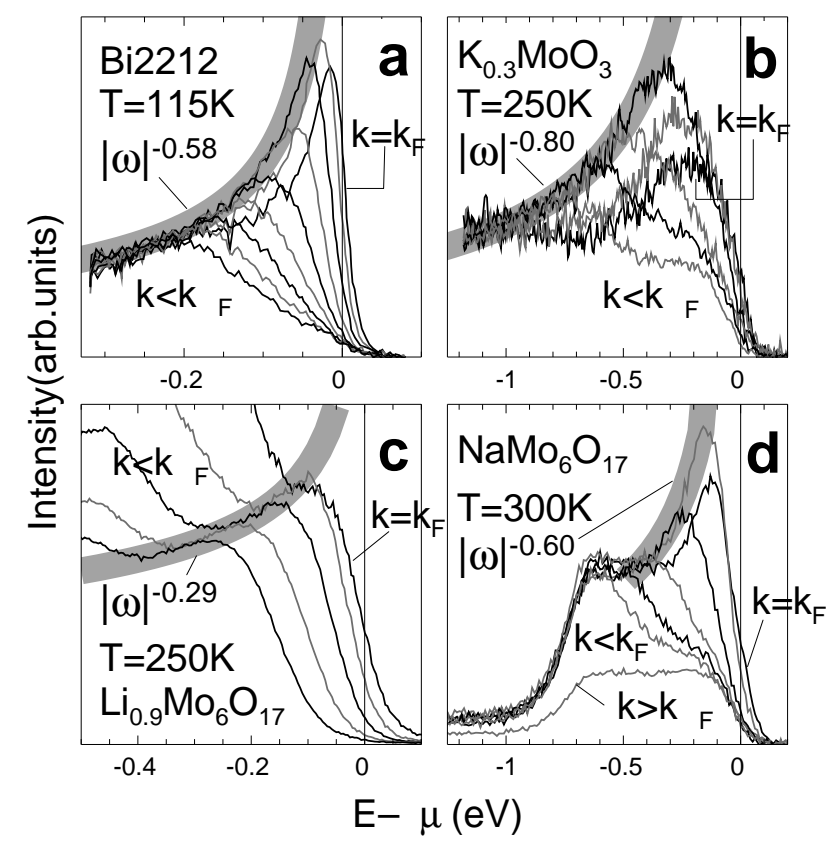

FIG. 3: Anderson-Ren plots for (a) the Bi2212 data taken along $\Gamma-\mathrm{Y}$ 22], (b) the quasi-1-d band for the $\mathrm{K}$ blue bronze 11], (c) the quasi-1-d band for the Li purple bronze [14], and (d) the hidden-1-d band crossing $\mu$ for the Na purple bronze. The data in (d) are shown again in Fig. 7 (a) as a stack plot with momentum value labels. For these plots, the intensity scale for individual curve was varied as a free parameter.

purple). These values show reasonable agreement with those found in Fig. 2 for the Li and Na purple bronzes, but not for the $\mathrm{K}$ blue bronze and Bi2212. For the $\mathrm{K}$ blue bronze this finding could signal that its spin pseudo-gap breaks the AR connection to $\alpha$. For Bi2212 we infer that the $\alpha=0$ result in Fig. 2 reflects an unobservably small energy range for power law behavior near $\mu$, as discussed above. The Li purple bronze ARPES data that we reported initially 12$]$ give an exponent of -0.2 (hence $\alpha=0.8)$ in reasonable agreement with the current data. We also verified that the tail exponent reported in Fig. 3] is modified only slightly to -0.35 , if we subtract out approximately the contribution from higher energy non$\mu$-crossing bands (see Fig. 4 caption). In short, our analysis distinguishes large $\alpha(>0.5)$ correlations found in the Li purple bronze and small $\alpha(<0.5)$ correlations found in the Na purple bronze and Bi2212, important for discussing the next signature.

Before proceeding, we mention that there is no known microscopic derivation for the AR line shape, yet [34]. It is easy to see that the LL fails because it produces line shapes for which the origin of the approximate power law is the moving position of the dispersive peak rather than the fixed $\mu$. This observation is intriguing, particularly in light of the otherwise excellent LL description of the Li purple bronze, as we will see now.

3. Two or More Objects in ARPES In the LL 
lineshape, two features disperse with velocities $\mathrm{v}_{\mathrm{c}}, \mathrm{v}_{\mathrm{s}}$, in contrast to the single quasi-particle peak of the FL. Depending on the magnitude of $\alpha$, the low binding energy spin feature is either a peak $(\alpha<0.5)$ or an edge $(\alpha>0.5)$ 36]. As we discussed and demonstrated before [12], by reason of its ideally $1-\mathrm{d}$ FS, linear band dispersion to at least $300 \mathrm{meV}$ below $\mu$, low transition temperature $(24 \mathrm{~K})$, and lack of a single particle gap opening at least down to $1 \mathrm{meV}$ in the low-T phase, the Li purple bronze is a unique candidate for a simple LL description, an $\alpha=0.9 \mathrm{TL}$ in particular. Fig. 4 shows our newest data set 14] compared with the new finite $T$ theory [15]. The same $\alpha$ value, 0.9 , that best simulates the amount of the weight at the $\mu$ crossing relative to the peak height also agrees nicely with the value obtained in Fig. 2 The $\mathrm{v}_{\mathrm{c}} / \mathrm{v}_{\mathrm{S}}$ value of 2 (and $\hbar \mathrm{v}_{\mathrm{c}}=4 \mathrm{eV} \AA$ ) is used as in Ref. [14], where we already noted that the improved angle resolution $\Delta \theta$ relative to that of our earliest work [12] gives better resolution of the spin edge and leads to $a v_{c} / v_{s}$ value changed from our early value of 5 . Overall, there is excellent agreement between the improved experiment and the improved theory. Fig. [5 shows that the quality of the agreement is definitely sensitive to the choice of $v_{c} / v_{s}$ and Fig. 6] shows the same for the choice of $\alpha$. Other features such as the general decrease of intensity as the peak approaches $\mu$ and the $\mu$ weight retraction after the peak has crossed $\mu$ are also reproduced with internal consistency by the theory. The same is true, of course, for the $\mu$ onset of the angle summed spectrum, and in this context we emphasize again the enormous difference between the angle summed spectra of the Fermi liquid reference material $\mathrm{TiTe}_{2}$ and of the $\mathrm{Li}$ purple bronze. The excellent agreement with the TL theory makes the Li purple bronze presently unique (apart from the AR tails which are nonetheless of clear NFL character) as an ARPES example of the TL line shape.

We generalize the idea of two dispersing objects in the TL line shape as follows. As shown in panel (d) of Fig. [3] and panel (a) of Fig. 7] the line shapes of the 2-d Na (or K) purple bronze [37] are surprisingly complex considering the simplicity and good agreement with band theory of the ARPES FS shown in Fig. 11 In particular, the lineshapes show two independent components, both the dispersing peaks that give the well defined FS and an equally large amount of $\mathbf{k}$-independent weight seen by itself in the $\mathbf{k}>\mathbf{k}_{\mathrm{F}}$ spectra. Because this weight is perfectly confined within the bandwidth it does not have an extrinsic origin, e.g. the inelastic scattering of photoelectrons. Charge disorder scattering giving $\mathbf{k}$-loss is likely, due to the well-known tendency for alkali atom deficiencies in the purple bronzes [19], but in a FL picture it is impossible to understand the fractionalized $\boldsymbol{k}$-loss seen here. The lack of apparent interaction between this weight and the dispersing peak, and its presence unreduced in amplitude after the peak crosses $\mu$, shows that it cannot be simply attributed to the incoherent part of

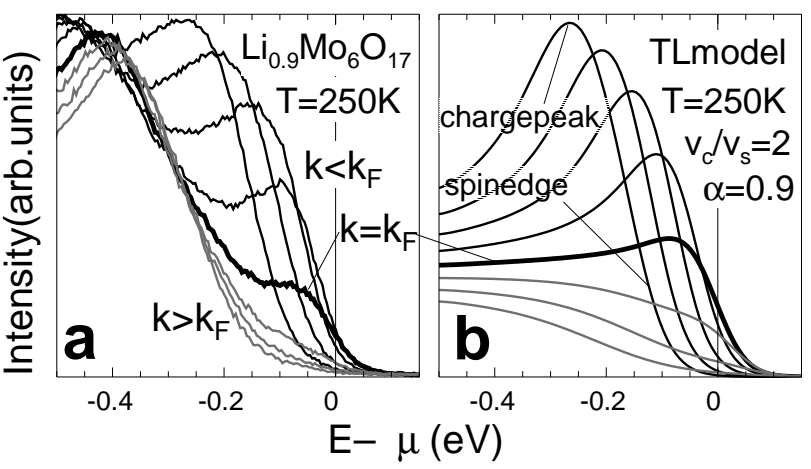

FIG. 4: High resolution angle resolved photoemission data for the Li purple bronze [14] taken along $\Gamma-Y$ (a) and TL model 15] simulation (b). The data show a single band crossing, due to the suppression of the other $\mu$-crossing band in this geometry [14]. In the data, the peaks with energy $<-0.3 \mathrm{eV}$ arise from a non- $\mu$-crossing band [1], which is excluded from the theoretical simulation. As the bottom curve (corresponding to $\mathbf{k} \gg \mathbf{k}_{\mathrm{F}}$ ) of panel (a) shows, the line shape of the non$\mu$-crossing band is a well confined peak, so the line shape of the $\mu$-crossing band can be observed essentially unhindered in an extended energy range, e.g. energies greater than its peak energy minus $\approx 0.1 \mathrm{eV}$ for $\mathbf{k} \leq \mathbf{k}_{\mathrm{F}}$. Experimental conditions $\left(T=250 \mathrm{~K}, \Delta E=49 \mathrm{meV}, \Delta \theta=0.36^{\circ}\right)$ are fully included in the simulation.

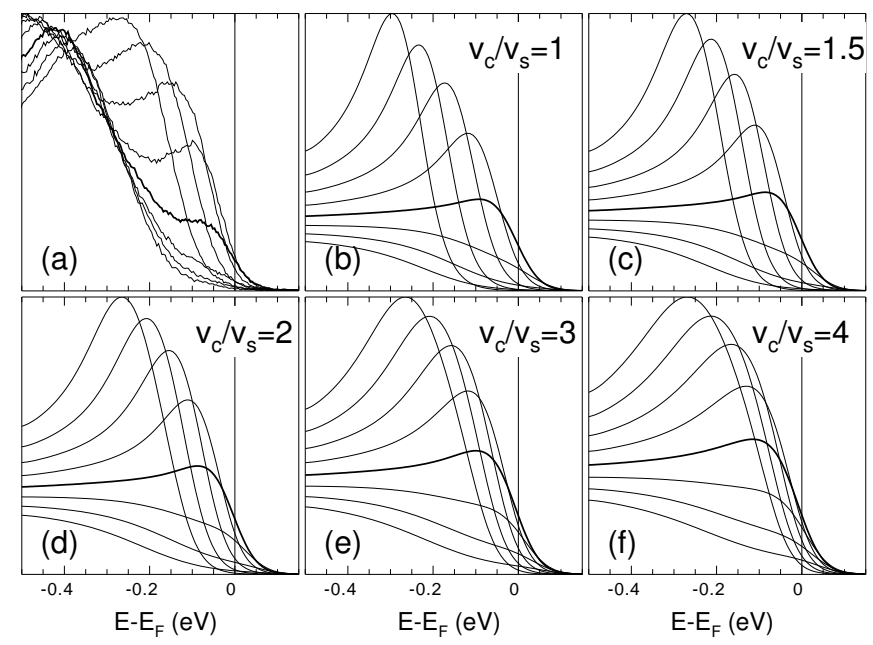

FIG. 5: TL model spectra from theory of Ref. [15] in panels (b) through (f) compared to Li purple bronze ARPES data of panel (a) and Fig.4(a) to show sensitivity of TL description of data to choice of ratio of velocities of holon peaks and spinon edges. Holon peak dispersion is held constant and matched to experimental peak dispersion for ease of comparison of spinon edge dispersions.

\section{a FL spectral function.}

We propose electron fractionalization, with badly scattered charge waves giving the $\mathbf{k}$-independent weight and spin waves, un-scattered because they do not see the charge disorder, dispersing to define the FS just as they do in the Li purple bronze. Here, the spin waves are 


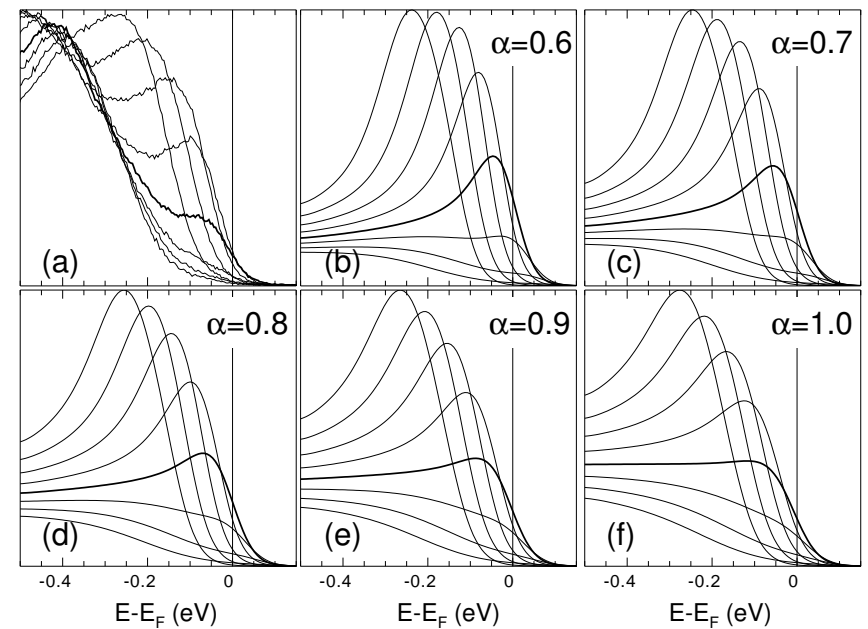

FIG. 6: TL model spectra from theory of Ref. [15] with $\mathrm{v}_{\mathrm{c}} / \mathrm{v}_{\mathrm{s}}$ value of 2 and various $\alpha$ in panels (b) through (f) compared to Li purple bronze ARPES data of panel (a) and Fig. 4 (a) to show sensitivity of TL description of data, with regard to the weight at $\mu$ and the broadness of the peak at $\mu$ crossing, to choice of $\alpha$.

peaks instead of edges because $\alpha<0.5$. Having now a clear example, augmented by other fractionalization signatures, confers much plausibility on the original suggestion [38] to us of this "melted holon" picture for Bi2212 (and other metallic cuprate) spectra. As seen in panel (b) of Fig. 77 these spectra also show, in addition to dispersing peaks, $\mathbf{k}$ independent weight 23] that is remarkably similar to that of the Na purple bronze except that the overlap with oxygen bands precludes knowing if it is also so neatly confined to the d-bandwidth. It is significant that the blue bronze has neither charge disorder nor kindependent weight. The 1-d Li purple bronze has charge disorder and again shows some $\mathbf{k}$-independent weight 11] although less than that of the Na purple bronze. Since the Li purple bronze spectra show both dispersing charge peaks and spin edges, this weight is perhaps some small portion of the charge peak weight but could signal the more intriguing possibility that fractionalization has occurred into three density excitations, a possibility which is actually known in theory [39], and is plausible in this material with two bands crossing $\mu$ together.

4. Sharp MDC, Broad EDC Orgad et al. 7] noted that the TL spectral functions show a sharp momentum distribution curve (MDC) at $\mu$ and a broad energy distribution curve (EDC) at $\mathbf{k}_{\mathrm{F}}$ while for the FL both are sharp. The mechanism for this contrast in the theory is the generic 1-d kinematic restrictions on momentum, but not on energy, for electron fractionalization into one or more kinds of dispersive density waves, as happens in, but is not restricted to, the TL (or LE) model. They reported this striking MDC/EDC contrast for cuprates, especially the static stripe system $\mathrm{La}_{1.25} \mathrm{Nd}_{0.6} \mathrm{Sr}_{0.15} \mathrm{CuO}_{4}$, but also the Bi2212. As seen in Fig. 8] our data for quasi-

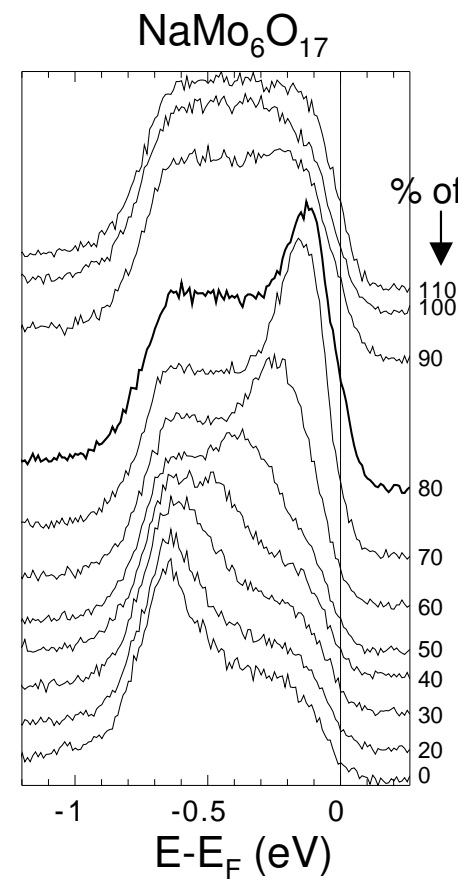

(a)

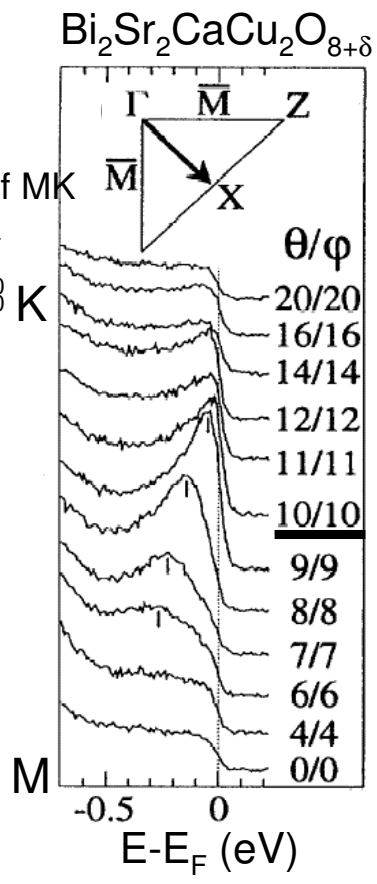

(b)
FIG. 7: ARPES lineshapes (a) along M-K for the quasi-2-d Na purple bronze [26] compared to those (b) along $\Gamma-\mathrm{X}$ for the Bi2212 [23].

1-d metals indeed show this contrast, a small momentum offset from the theory for the Li purple bronze 40. notwithstanding. So does the Na purple bronze data of Fig. [7 (also see [37]) once we recognize the $\mathbf{k}$-independent (badly scattered charge wave) weight as part of the intrinsic spectrum. It is precisely the $\mathbf{k}$-independent weight in the Bi2212 spectra that enabled the identification made by Orgad et al. 7] of this signature in Bi2212 and our discussion here enables us to offer an explicit proposal as to the origin of the weight within a fractionalization picture. Strong arguments that this weight is intrinsic to the spectral function have been given previously in Refs. [41] and [42].

Note that this MDC/EDC contrast requires at least moderately large $\alpha$ [], further supporting our conclusion of a non-zero but small $\alpha$ for Bi2212 from the combined analysis of signatures 1 and 2 . Note also that $\mathrm{v}_{\mathrm{s}}$ is used to scale the momentum axis of the MDC plots. While it means the spin wave velocity for the TL model (and therefore for the Li purple bronze), its generic meaning is the smallest velocity of density waves involved, and for the poorly understood spin-gapped $\mathrm{K}$ blue bronze we use the value of $1 \mathrm{eV} \AA$ to make its MDC width similar to that of the Li purple bronze. 

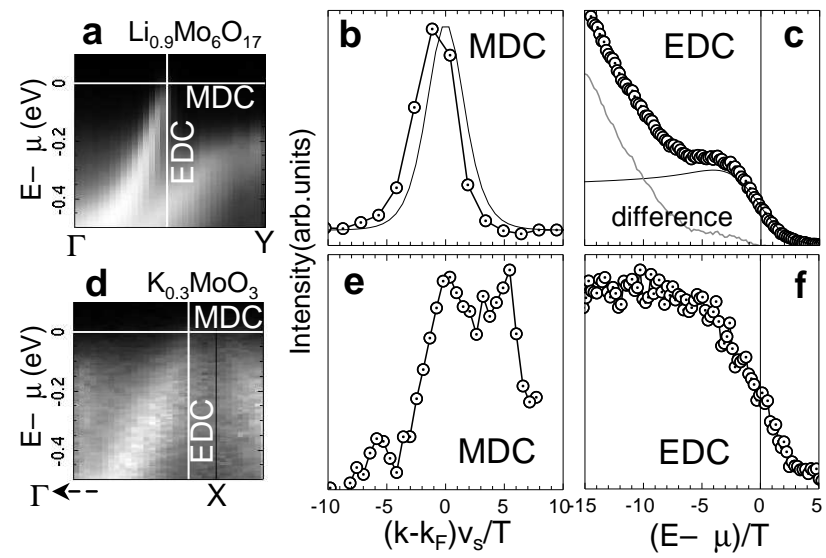

FIG. 8: ARPES intensity map for the Li purple bronze (a), and its MDC (b) and EDC (c) cuts, and similar plots for the $\mathrm{K}$ blue bronze ( $\mathrm{d}$, e, and $\mathrm{f}$ ). In (b) and (c), theory curves of the TL model, as used in Fig. 4 are shown as thin solid lines. In (c), the difference between the data and the theory is shown as a gray solid line, representing the non- $\mu$-crossing band. The $\mathrm{K}$ blue bronze has three crossings showing as three peaks in the MDC, and here we focus on the central crossing, but others lead to the same conclusion.

\section{SUMMARY}

To summarize, our "score card" representation of the electron fractionalization signatures is the following: $\mathrm{Li}$ purple bronze $(1,2,3,4)$, K blue bronze $(1,2,4)$, Na purple bronze $(1,2,3,4)$, Bi2212 $(2,3,4)$. Our electron fractionalization signatures are ubiquitous in the sense that each of the examined materials shows at least three signatures. The Li purple bronze displays all four and as such is a quasi-1-d LL paradigm. The missing signature in the quasi-1-d K-blue bronze can be attributed to complexities of a spin gap and its incipient CDW ordering. Neither of these materials seems to require the ideas of Ref. [43]. It is especially notable that the last two of our materials are quasi-2-d and that the $\mathrm{Na}$ purple bronze displays all four signatures within the framework of our "melted holon" interpretation. Like the Na purple bronze, Bi2212 could well be fractionalized with nonzero $\alpha<0.5$ and badly scattered charge waves, but with either $\alpha$ or the energy range of power law behavior too small to detect in the $\mu$ onset of its angle integrated spectrum. We note that the $\mathbf{k}$-independent spectral weight of both materials demands some explanation outside of the FL picture. We argue here that the $\mathrm{Na}$ purple bronze is a kind of Rosetta stone that enables the recognition of the effects of disorder on fractionalization in the Bi2212 spectrum.

Overall, it is remarkable that all these systems display common, generic signatures within our generalized electron fractionalization scheme regardless of their different, and often not fully understood, low $T$ physics, and different global dimensionalities. Our findings are a strong hint of a bigger picture in which fractionalization plays a central role. In closing we mention that another important general fractionalization signature that is implicit in our use of the theory of Ref. 15] is that of quantum critical scaling in the energy and temperature dependence of ARPES lineshapes. Such scaling has already been reported for Bi2212 lineshapes [44] and our plans for the future include such studies for the other materials we have discussed.

GHG and JWA acknowledge useful discussions with S.A. Kivelson. For the data of Fig. B(d), we are indebted to J. Marcus and C. Schlenker, to W.P. Ellis, R. Claessen and F. Reinert, and to Z.-X. Shen for providing samples, for participation in the experiments, and for use of his end-station, respectively. This work was supported by the U.S. NSF grant No. DMR-99-71611 and the U.S. DoE contract No. DE-FG02-90ER45416 at U. Mich.

$\dagger$ Current address: Lawrence Berkeley National Laboratory, MS 7-100, 1 Cyclotron Road, Berkeley, CA 94720

[1] L.D. Landau, Sov. Phys. JETP 30, 1058 (1956).

[2] F.D.M. Haldane, J. Phys. C 14, 2585 (1981).

[3] S. Tomonaga, Prog. Theor. Phys. 5, 544 (1950).

[4] J. M. Luttinger, J. Math Phys. 4, 1154 (1963).

[5] P.W. Anderson, Physics Today 50, 42 (1997).

[6] S.A. Kivelson, Synthetic Metals 125, 99 (2002).

[7] D. Orgad et al., Phys. Rev. Lett. 86, 4362 (2001).

[8] A. Luther and V. J. Emery, Phys. Rev. Lett. 33, 589 (1974).

[9] B. Dardel et al., Phys. Rev. Lett. 67, 3144 (1991).

[10] M. Grioni and J. Voit, in Electron spectroscopies applied to low-dimensional materials (eds. H.P. Hughes and H.I. Starnberg) p. 209 (Kluwer, Dordrecht, 2000).

[11] G.-H. Gweon et al., J. Elec. Spectro. Rel. Phenom. 117118, 481 (2001).

[12] J.D. Denlinger et al., Phys. Rev. Lett. 82, 2540 (1999).

[13] G.-H. Gweon et al., Phys. Rev. Lett. 85, 3985 (2000).

[14] G.-H. Gweon et al., Physica B, in print.

[15] D. Orgad, Philos. Mag. B 81, 377 (2001).

[16] J.P. Pouget, private commmunication.

[17] L. Degiorgi et al., Phys. Rev. B 38, 5821 (1988).

[18] M. Greenblatt et al., Solid State Commun. 51, 671 (1984).

[19] C. Schlenker, ed. Low-Dimensional Electronic Properties of Molybdenum Bronzes and Oxides (Kluwer Academic Publishers, Bordrecht/Boston/London, 1989).

[20] M. H. Whangbo et al., Science 252, 96 (1991).

[21] G.-H. Gweon et al., Phys. Rev. B 55, R13353 (1997).

[22] A. Kaminski et al., Phys. Rev. Lett. 86, 1070 (2001).

[23] D.S. Dessau et al., Phys. Rev. Lett. 71, 2781 (1993).

[24] E. W. Carlson et al., Phys. Rev. B 62, 3422 (2000).

[25] R.H. McKenzie and D. Scarratt, Phys. Rev. B 54 R12709 (1996).

[26] The data in Fig. 3(d) were taken at Beamline 5 of the Stanford Synchrotron Radiation Laboratory, with $h \nu=$ $22.4 \mathrm{eV}, \Delta E=43 \mathrm{meV}$ and $\Delta \theta=2^{\circ}$.

[27] R. Claessen et al. Phys. Rev. Lett. 69, 808 (1992).

[28] R. Claessen et al. Phys. Rev. B 54, 2453 (1996).

[29] L. Perfetti et al. Phys. Rev. B 64, 115102 (2001). 
[30] The data near the 2 -d BZ edge (i.e. near the $(\pi, 0)$ point) [A. Kaminski et al., private communication.] yield $\alpha=0.1$, signaling some $\mathbf{k}$ dependence of $\alpha$. The small band dispersion near $(\pi, 0)$ precludes an AR analysis.

[31] K. Penc, F. Mila, and H. Shiba, Phys. Rev. Lett. 75, 894 (1995).

[32] D.C. Johnston, Phys. Rev. Lett. 52, 2049 (1984).

[33] P.W. Anderson and Y. Ren, in High Temperature Superconductivity (eds. K. S. Bedell et al.) p.3 (AddisonWesley, Redwood City, 1990).

[34] P.W. Anderson has suggested to us that the AR lineshape might obtain for the "melted holon" scenario discussed for signature number 3 .

[35] T. Valla et al., Phys. Rev. Lett. 83, 2085 (1999).

[36] V. Meden and K. Schönhammer, Phys. Rev. B 46, 15753 (1992); J. Voit, Phys. Rev. B 47, 6740 (1993).

[37] G.-H. Gweon et al., J. Phys. Condens. Matter 8, 9923
(1996)

[38] P.W. Anderson, private communication, 1995.

[39] L. Balents and M. P. A. Fisher, Phys. Rev. B 53, 12133 (1996).

[40] While we do not have a firm explanation for this small effect, a slightly larger experimental MDC width and the matrix element modulation that gives a strong suppression of weights for $\mathbf{k}>\mathbf{k}_{\mathrm{F}}$ would be a likely explanation.

[41] G.A. Sawatzky, in High Temperature Superconductivity (eds. K. S. Bedell et al.) p.297 (Addison-Wesley, Redwood City, 1990).

[42] L. Z. Liu, R. O. Anderson and J.W. Allen, J. Phys. Chem. Sol. 52, 1473 (1991)

[43] P. Starowicz et al., Phys. Rev. Lett. 89, 256402 (2002).

[44] T. Valla et al., Science 285, 2110 (1999). 